\title{
Multidrug-Resistant Tuberculosis in the Democratic Republic of Congo: Analysis of Continuous Surveillance Data from 2007 to 2016
}

\author{
Serge Bisuta-Fueza ${ }^{*}$, Jean Marie Kayembe-Ntumba1, Marie-Jose Kabedi-Bajani2, \\ Pascale Mulomba Sabwe ${ }^{3}$, Hippolyte Situakibanza-Nani Tuma ${ }^{4}$, Jean-Pierre Simelo ${ }^{3}$, \\ Ernest Sumaili-Kiswaya ${ }^{5}$, John Ditekemena-Dinanga ${ }^{6}$, Patrick Kayembe-Kalambayi ${ }^{7}$ \\ ${ }^{1}$ Pneumology Unit, University Hospital of Kinshasa, University of Kinshasa, Kinshasa, Democratic Republic of Congo \\ ${ }^{2}$ Department of Microbiology, Medical Biology Department, University of Kinshasa, Kinshasa, Democratic Republic of Congo \\ ${ }^{3}$ National Tuberculosis Program, Ministry of Public Health, Kinshasa, Democratic Republic of Congo \\ ${ }^{4}$ Infectious Diseases Unit, Department of Internal Medicine, University of Kinshasa, Kinshasa, Democratic Republic of Congo \\ ${ }^{5}$ Physiology Unit, Department of Internal Medicine, University of Kinshasa, Kinshasa, Democratic Republic of Congo \\ ${ }^{6}$ Department of Community Health, Kinshasa School of Public Health, University of Kinshasa, Kinshasa, Democratic Republic of \\ Congo \\ ${ }^{7}$ Department of Epidemiology \& Biostatistics, Kinshasa School of Public Health, University of Kinshasa, Kinshasa, Democratic \\ Republic of Congo \\ Email: ^Serge.bisuta@unikin.ac.cd
}

How to cite this paper: Bisuta-Fueza, S., Kayembe-Ntumba, J.M., Kabedi-Bajani, M.-J., Sabwe, P.M., Tuma, H.S.-N., Simelo, J.-P., Sumaili-Kiswaya, E., Ditekemena-Dinanga, J. and Kayembe-Kalambayi, P. (2019) Multidrug-Resistant Tuberculosis in the Democratic Republic of Congo: Analysis of Continuous Surveillance Data from 2007 to 2016. Journal of Tuberculosis Research, 7 , 25-44.

https://doi.org/10.4236/jtr.2019.71004

Received: March 1, 2019

Accepted: March 25, 2019

Published: March 28, 2019

Copyright $\odot 2019$ by author(s) and Scientific Research Publishing Inc. This work is licensed under the Creative Commons Attribution International License (CC BY 4.0).

http://creativecommons.org/licenses/by/4.0/ cC) (i) Open Access

\begin{abstract}
Background: For countries with limited resources such as the Democratic Republic of the Congo (DRC), the diagnosis of Multidrug-resistant tuberculosis (MDR-TB) is still insufficient. The MDR-TB identification is done primarily among at-risk groups. The knowledge of the true extent of the MDR-TB remains a major challenge. This study tries to determine the proportion of MDR-TB in each group of presumptive MDR-TB patients and to identify some associated factors. Methods: This is an analysis of the DRC surveillance between 2007 and 2016. The proportions were expressed in Percentage. The logistic regression permits to identify the associated factors with the RR-/MDR-TB with adjusted Odds-ratio and 95\% CI. Significance defined as $\mathrm{p} \leq 0.05$. Results: Overall, $83 \%$ (5407/6512) of the MDR-TB presumptive cases had each a TB test. $86.5 \%$ (4676/5407) had each a culture and drug sensitive testing (DST) on solid medium, and 24.3\% (1312/5407) had performed an Xpert MTB/RIF test. The proportion of those with at least one first-line drug resistance was 59.3\% [95\% CI 57.2 - 61.4] among which 50.1\%, [95\% CI 47.9 - 52.3] for the isoniazid, $45.6 \%$ [95\% CI 43.4 - 47.8] for the rifampicin, 49.9\% [95\% CI 47.8 - 52.1] for ethambutol and 35.8\% [95\% CI 33.7 - 37.9] for
\end{abstract}


streptomycin. The confirmation of MDR-TB was 42.8\% [95\% CI 38.4 - 47.8]. Combining both tests, the proportion of RR-/MDR-TB was $49.6 \%$ [95\% CI 47.9 - 51.4] for all presumptives. This proportion was $60.0 \%$ for failures, $40.7 \%$ for relapses and $34.7 \%$ for defaulters. Associated factors with the diagnosis of MDR-TB were: aged less than 35 years; prior treatment failure; defaulters; the delay between the collection of sputum and the test completion. Conclusion: The proportion of RR-/MDR-TB among the presumptives has been higher than those estimated generally. The National tuberculosis programme (NTP) should improve patient follow-up to reduce TB treatment failures and defaulting. Moreover, while increasing the use of molecular tests, they should reduce sample delivery times when they use culture and DST concomitantly.

\section{Keywords}

Multidrug-Resistant Tuberculosis, Resistance to Rifampicin, Factors Associated, DR Congo

\section{Introduction}

MDR-TB is the tuberculosis with resistant bacilli to isoniazid (INH) and rifampicin (RMP), the two most powerful antituberculosis drugs. The incorrect use of drugs, the inefficient uptake of therapeutic regimes and the premature discontinuation of treatment cause drug-resistances (DR-TB) that are likely to be transmitted to the population, especially in places with a strong promiscuity, such as prisons, hospitals, orphanages and some dwellings [1].

In contrast to drug-susceptible $\mathrm{TB}$, the diagnosis of $\mathrm{MDR}-\mathrm{TB}$ requires more complex laboratory investigations than simple microscopy [2]. The resistance profile is obtained either by the sensitivity test after so-called "traditional" methods, such as culture on liquid or solid medium, whose average duration varies from 2 to 8 weeks. Or you can use the rapid diagnosis or molecular diagnostics (RMD), the most common of which is the Xpert MTB/RIF test, which detects M. tuberculosis (MTB) and rifampicin resistance (RR) with greater sensitivity [3] [4]. According to the World Health Organization (WHO) report of 2017, the proportion of MDR-TB cases among new patients is estimated at $4.1 \%$ [95\% CI: $2.8 \%-5.3 \%$ ] and 19\% [95\% CI: 9.8\% - 27\%] among the patients already treated, for the entire world, about $600,000(540,000$ - 660,000) cases [5] [6]. The MDR-TB most affected regions are respectively Central Europe and the countries of the former Soviet Union, Southeast Asia (China and India) and sub-Saharan Africa, and particularly the southernmost [5].

Under the best conditions, all tuberculosis patients should have a profile of $\mathrm{DR}-\mathrm{TB}$ as is the case in developed and advanced countries; this allows to prescribe personalized therapy and to obtain real TB prevalence drug-resistant TB [1] [6] [7] [8].

Africa, which contains only $12 \%$ of the world's population, has $25 \%$ of TB 
cases [5] [7] [9]. The number of MDR-TB cases in Africa is estimated at 93,000 (range: 81,000 - 106,000) cases, or $15 \%$ of the global burdens based on estimations with $2.7 \%$ [95\% CI: 2.0 - 3.5] are cases of MDR-TB among new patients, and 14\% [95\% CI: 8.4 - 20] are cases of MDR-TB among previously treated cases. In the continent, the burden is generated by the Republic of South Africa (RSA), Nigeria, Ethiopia, the DRC and other countries in the southern region that have a high prevalence of HIV infection [5] [10].

In 2015 and 2016, the number of TB cases reported in the DRC was respectively 120,508 and 132,515 , of which $64 \%$ were new patients with bacteriologically confirmed pulmonary tuberculosis [5] [10] [11]. The number of patients in retreatment for these two years was 5399 and 5490, representing around $4 \%$ of all the TB cases. The latter group consists of 3 types of patients with a higher risk of developing MDR-TB: relapses (incident cases), failures to initial treatment and retreatment after being defaulters [11].

For poor countries such as the DRC, where access to the diagnosis of MDR-TB is still insufficient, the MDR-TB identification is done primarily among the at-risk groups [1] [6] [7]. The knowledge of the extent of the disease remains a major challenge. The recent DRC prevalence survey found a prevalence of $2.2 \%$ of MDR-TB among new patients with bacteriologically confirmed TB (NP TP +) and 10\% among previously treated cases [5]. That kind of survey is actually effective only if it is performed every 3 to 5 years. The estimate of this magnitude can also be deduced by a routine monitoring of at-risk groups [6] [12]. Groups of presumptives MDR- TB (which includes retreatment cases) are defined according to the period when the sputum persistence is detected by microscopy during treatment administered under surveillance. The proportion of MDR-TB and the risk are different from one type to another; it is useful to determine it [4] [7] [13]. National guidelines in the DRC agree with these WHO recommendations [14] [15]. However, previous studies in this area have focused either on the new patient group [6] [10] or on the risk factors [16] [17]. There is currently no long-term study that analyzes the results of MDR-TB surveillance since the integration of MDR-TB control.

The MDR-TB surveillance is a fundamental part of the TB control strategy, or even its elimination. The objective of this study is to determine the proportion of MDR-TB in each group of presumptive MDR-TB patients in the DRC and to identify the associated factors to MDR-TB.

\section{Methods}

\subsection{Type, Site and Period of the Study}

This is an analysis of 10-year-surveillance data. The MDR-TB presumptive patients came from all the 11 provinces of the DRC during the period from January 1, 2007 to December 31, 2016 (Figure 1).

All MDR-TB presumptive patients were included. They are defined in the MDR-TB management guide in the DRC [6] [7]. Their sputum samples were sent to the Kinshasa National Reference Laboratory (NRL) for diagnosis. 


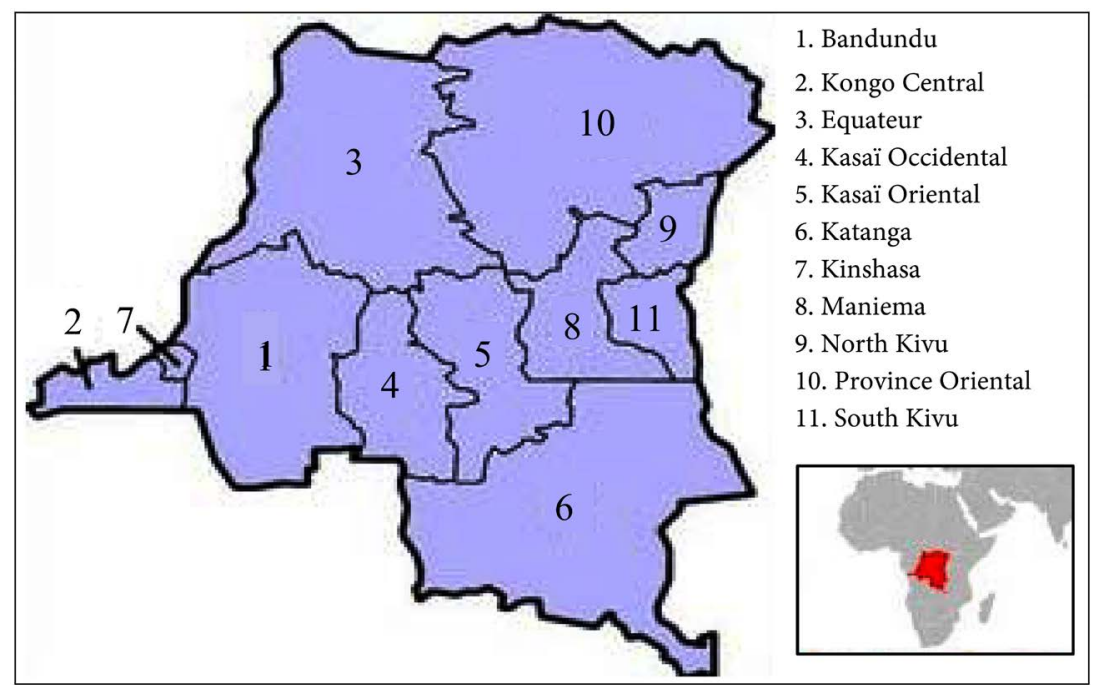

Figure 1. Map of the DRC showing the 11 former provinces until 2015 as used in this study. And at the bottom right is the DRC in Africa.

The collection, the packaging and the transportation of specimens were done at health centers whose staffs were trained in the procedures for collecting and packaging specimen's. For each presumptive, two sputum samples were collected and shipped to the NRL. For remote structures samples were pretreated with a preservative (cetyl pyriridium chloride (CPC) at $1 \%$ ), and for the closely structures, the samples were kept cool. The biological material was kept in hermetically sealed isothermal boxes complying with ISO safety standards [18] [19].

\subsection{Bacteriological Tests Carried out}

Two tests were used, the first phenotypic by culture and the DST on solid medium of Löwenstein-Jensen (proportion technique) and the second molecular by GeneXpert MTB/RIF to detect MTB. Antibiotic or susceptibility testing was performed for the four first-line anti-TB drugs isoniazid (INH, H), rifampicin (RMP, R), ethambutol (EMB, E) and streptomycin (SM, S) [8] [11] [15] [18].

For Xpert testing, sputum specimens were tested as recommended by the manufacturer (Cepheid), adopted by WHO and included in the national guidelines and the test has to determine resistance to rifampicin (RR) [2] [3] [7].

\subsection{Data Sources}

Data were collected from the NRL of the NTP, at the provincial coordination and MDR-TB health centers.

The information gathered from the examination application documents allowed for the categorization of the suspects as discussed above. At the laboratory level all information and results were centralized in an electronic register. The quality control of these data was applied by comparing the data between the different sources: laboratory, treatment centers and files from the provincial coordination. 


\subsection{Definition of Concepts}

The definitions used for this study have been developed in accordance with the WHO updates, which are included in the national technical guide [7] [20] [21]. Table 1 provides the operational definitions of presumptive types and the results of standard sensitivity tests and molecular tests.

\subsection{Variables of Interest and Data Analysis}

The essential data consisted of age, gender, health zone and the center where the patient was followed, the province of residence. The different dates, sputum collection, their arrival at the laboratory, the type of test performed the results of the DST on the 4-drugs (RMP, INH, EMB and SM) and the Xpert-MTB/RIF test results when they were available. Patients were grouped in 2-year period corresponding to the periodicity of adaptations of MDR-TB case-finding guidelines.

The sampling dates, the arrival of the sample at the laboratory and the execution of the test allowed us to calculate the deadlines. The averages of the numeric variables and the standard deviation (SD) were calculated. The proportions were sought expressed in Percentage with 95\% CI.

The comparison of the subpopulations defined by the periods was made using the Chi-square test of homogeneity on the age groups. The ANOVA test for population inequality was applied for the calculation of the age averages for the 5 periods. The $\mathrm{Z}$ score for the comparison of the proportions was used with a significant threshold of $5 \%$. The logistic regression in bivariate and multivariate analysis was used to assess the associations between the potentials factors and the MDR-TB. Odds-Ratio (OR) with the $95 \%$ confidence interval (CI) was used to assess the level of association. Significance was defined as $\mathrm{p} \leq 0.05$.

\subsection{Ethical Considerations}

The different strategies were discussed and approved in a working committee including the staff of National tuberculosis Program (NTP) and TB partners. Field actors were trained in cascade and then recycled as guidelines were changed. Respect for the patient's person and confidentiality were taken into account. All persons diagnosed with MDR-TB were put under standardized treatment with second-line drugs according to the National Tuberculosis Program guidelines. The study as analysis of surveillance was approved by the Ethics Committee of the School of Public Health of the University of Kinshasa $\left(\mathrm{N}^{\circ}\right.$ ESP/CE/066/2018).

\section{Results}

The number of patients received for a laboratory test during the period was 8008 patients, of whom $6512(81.3 \%)$ for a drug resistance study, of whom 5407 (83.0\%) had actually performed culture tests. and/or Xpert MTB/RIF with available results. Lab results were not found for 1105 (17.0\%) patients (Figure 2). 
Table 1. Definitions used in the study.

\begin{tabular}{l}
\hline Category $\quad$ Definition \\
$\begin{array}{l}\text { Type of presumptive } \\
\text { and code used }\end{array}$
\end{tabular}

F1: failure of initial treatment

F2: failure of retreatment

F3: failure of a $3^{\text {rd }}$ treatment

R1: relapse after initial treatment completed at the end of initial treatment, and are now diagnosed with a recurrent episode of TB.

Previously treated for TB, were declared cured or treatment

$\mathrm{R} 2$ : relapse after retreatment

R3: relapse after 3 or more treatment

OT: other previously treated completed at the end of retreatment, and are now diagnosed with a recurrent episode of TB.

Previously treated for TB, were declared cured or treatment completed at the end of a $3^{\mathrm{RD}}$ treatment, and are now diagnosed with a recurrent episode of TB.

previously treated for TB but whose outcome after their most recent course of treatment is unknown or undocumented

D1: return after default to initial Previously treated for TB and were recognized lost to follow-up treatment during the initial treatment.

D2: return after default to retreatment

D3: return after default to a $3^{\text {rd }}$ treatment

C2: Sputum smears positive at the end of intensive phase of initial treatment

NC: case of MDR-TB patient contact

HIV: Person living with HIV Aids

\section{Results of sensitivity test}

Monoresistance

Polyresistance

Multi drug resistant tuberculosis (MDR - TB)

Tuberculosis in susceptible bacilli

Resistance to rifampicin (RR)

Multi drug resistant and resistant to rifampicin (RR-/MDR-TB)
Previously treated for TB and were recognized lost to follow-up during the retreatment.

Previously treated for TB and were recognized lost to follow-up during a $3^{\mathrm{RD}}$ or more treatment. This is at least a third episode.

Patient who remain sputum-smear positive at 2 month of initial treatment.

Exposure to a known RR-/MDR-TB case. Often family contact, with 2 months at least have lived closely (or promiscuous) with a patient with RR-/MDR-TB.

Person living with HIV Aids

Resistance to a TB only of first-line drug other than Rifampicin

Resistance to more than one anti-TB first-line drug without combining rifampicin and isoniazid.

Combined resistance to rifampicin and isoniazid with or without others.

Sensitivity to rifampicin and isoniazid

Resistance to rifampicin detected using genotypic, phenotypic methods with or without resistance to other drugs.

Sets of the phenotypic and genotypic test-confirmed cases of $\mathrm{RR}-/ \mathrm{MDR}-\mathrm{TB}$. This includes any form of resistance to rifampicin, the patterns, the polyrésistance and the MDR-TB. 


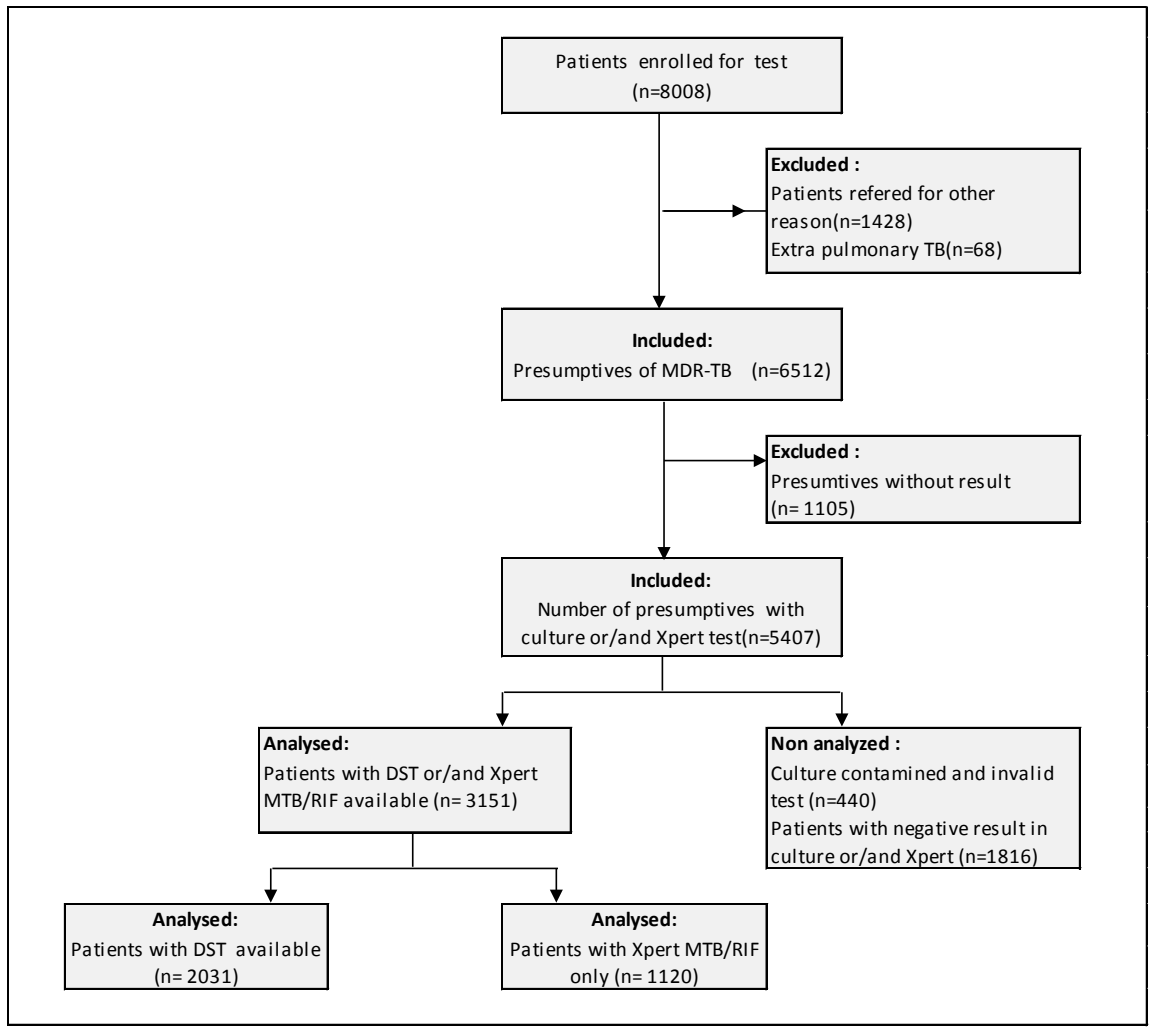

Figure 2. Flow diagram of the analysis of the results. Legend. Analyzed means group of patients in which MDR-TB/RR proportion was calculated. DST: drug sensitivity test.

\subsection{Sociodemographic Parameters of the Study Population}

The mean age was 37.04 years $(\mathrm{SD}=13.54)$, women 34.92 years $(\mathrm{SD}=13.47)$ and men $38.17 \pm 13.47(\mathrm{p}<0.001)$. The sex ratio $\mathrm{H} / \mathrm{F}$ was $1.46(146 \mathrm{H} / 100 \mathrm{~F})$.

Two thirds of the patients $4402 / 6512(67.6 \%)$ were in the age groups of 25 to 54 years.

The origin of patients by their province of origin is shown in Table 2 .

\subsection{Enrollment of Presumptive Per Period}

The inequality of the populations sought by considering the periods as a subgroup was not demonstrated, $\mathrm{p}=0.424$ (ANOVA) and the homogeneity of the sample according to the age groups was confirmed, $\mathrm{p}=0.3863$ (Chi square of homogeneity).

Enrollment of patients by category is reproduced in Figure 3.

Period 1: During the first period the majority of the suspects are F2 (76.3\%), $21.0 \%$ of the patients whose antecedents are not known and $2 \%$ of the cases of failure after the 3 rd regime received.

In the $2^{\text {nd }}$ period: The number of patients referred for MDR-TB research doubled, F2 increased from 454 to 501 and accounted for $49 \%$ of all patients. This period saw $82(8.0 \%)$ patients in retreatment who were divided into 44 (4.30\%) for the F1, 38 (3.71\%) for the R1. Patients relapsed after retreatment (R2) were $89(8.70 \%)$. 
Table 2. Distribution of patients by province and place of rural or urban origin.

\begin{tabular}{cccc}
\hline \multirow{2}{*}{ Province } & \multicolumn{2}{c}{ Middle of origin } & \multirow{2}{*}{ Total (\%) } \\
\cline { 2 - 3 } Bandundu & rural & Urban & \\
\hline Kongo Central & $142(11.7)$ & $65(1.2)$ & $207(3.2)$ \\
Equateur & $68(5.6)$ & $217(4.1)$ & $285(4.4)$ \\
Kasai Occidental & $150(12.3)$ & $146(2.8)$ & $296(4.5)$ \\
Kasai Oriental & $100(8.2)$ & $122(2.3)$ & $222(3.4)$ \\
Katanga & $148(12.2)$ & $245(4.6)$ & $393(6.0)$ \\
Kinshasa & $334(27.5)$ & $621(11.7)$ & $955(14.7)$ \\
Maniema & N/A & $3624(68.4)$ & $3624(55.7)$ \\
North Kivu & $66(5.4)$ & $23(0.4)$ & $89(1.4)$ \\
Province Oriental & $21(1.7)$ & $18(0.3)$ & $39(0.6)$ \\
South Kivu & $151(12.4)$ & $187(3.5)$ & $338(5.2)$ \\
TOTAL (\%) & $36(3.0)$ & $28(0.5)$ & $64(1.0)$ \\
\hline
\end{tabular}

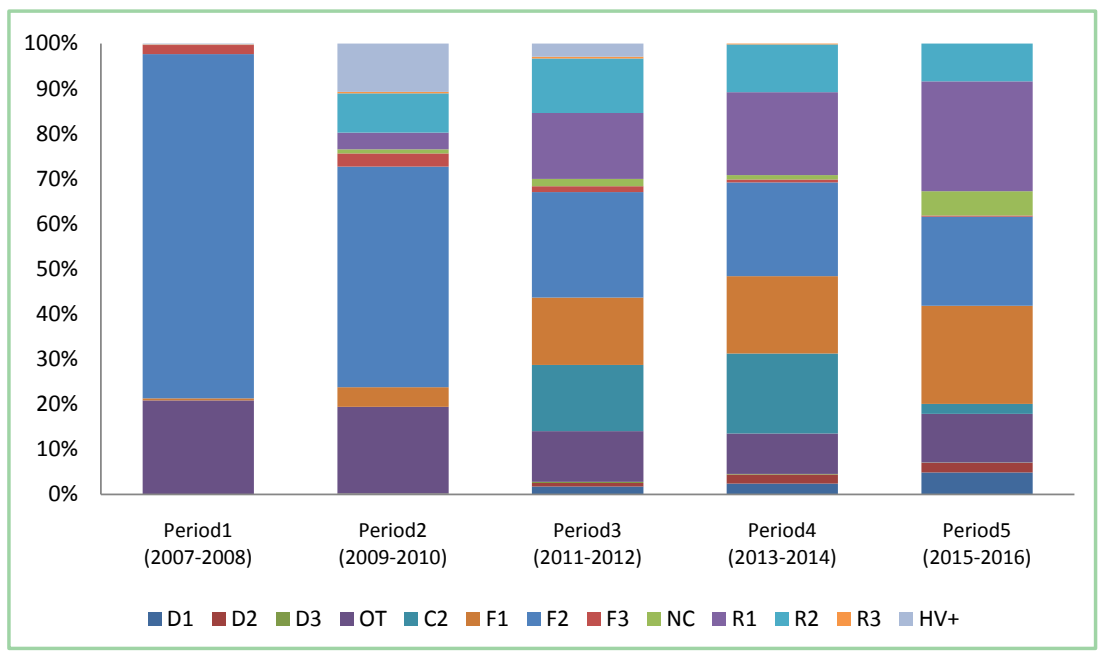

Figure 3. Enrollment charts of different types of presumptive MDR-TB by period.

In the $3^{\text {rd }}$ period: $40(2.8 \%)$ patients who had previously been reported lost to follow-up and had a positive Pap smear, 25 (1.7\%) after one episode and the rest with two or more treatment series. There were 212 patients with positive smears at the end of the intensive phase. The absolute number of presumptives retreatment doubled for each of the subcategories compared to the previous period, 216 (14.94\%) for the F1, 211 (14.59\%) for the R1 and 175 (12.10\%) for the R2.

During the $4^{\text {th }}$ and $5^{\text {th }}$ periods, there was a $48.54 \%$ increase in the number of patients whose samples were sent to the laboratory, $812(37.0 \%)$ for the cases retreatment distributed in 369 (17.2\%), 395 (18.4\%) and 52 (2.4\%) respectively for the F1, R1 and D1. The variation is significant between the last two periods concerning the proportion of the cases in retreatment $(\mathrm{R} 1+\mathrm{F} 1+\mathrm{D} 1)$ is $816 / 2148$ 
$(38.0 \%)$ for the $4^{\text {th }}$ period and $663 / 1300(51.0 \%)$ for the $5^{\text {th }}$ period, $(\mathrm{Z}$ score $=$ 7.4814, $\mathrm{p}<0.001)$.

The number of relapsed patients $(\mathrm{R} 1+\mathrm{R} 2)$ or incident cases previously treated decreased in number but not in proportion to the previous period, from $622(29.0 \%)$ to $426(32.8 \%)$, (Z score $=2.3586, \mathrm{p}=0.01828)$.

The proportion of F2 more than $3 / 4$ in the $1^{\text {st }}$ period has decreased and only represents $1 / 5^{\text {th }}$ of all cases $454 / 595$ (76.3\%) to $275 / 1300$ (19.8\%), (Chi 2 trends, $\mathrm{p}<0.001)$.

Compared with the history of tuberculosis among the 6512 patients, the number was 2016 (31.0\%) for those who had been diagnosed and treated once, 2680 (41.1\%) for those who had experienced 2 episodes and 101 (1.6\%) for those who had experienced 3 or more episodes of TB treated. Regarding the treatment results in the last episode the number was 2990 (46.0\%) for failures, 1575 (24.2\%) for relapses and $232(3.6 \%)$ for former defaulters.

\subsection{Laboratory Results}

Table 3 gives us the availability of the tests performed and the results of the culture on solid medium.

The evaluation recorded a gradual reduction in the availability of standard exams (culture and DST). The GeneXpert MTB/RIF test was effective during the 4 th period.

Overall (5407/6512) $83 \%$ of the presumptives had a test performed and the results were available, 4676 (71.8\%) had a culture result on Löwenstein-Jensen medium. The Xpert MTB /RIF test was performed for 1312 patients. The proportion of patients with GeneXpert MTB/RIF for the last two periods was 37\% or $1276 / 3448$ patients.

At the $3^{\text {rd }}$ and $4^{\text {th }}$ periods the availability in culture is respectively $57.1 \%$ and $53.4 \%$.

Table 3 shows detection rate of MTB by culture at $46.8 \%$ (range $35.1 \%$ $73.1 \%)$ and $387(8.3 \%)$ samples were contaminated, with a maximum of $16.3 \%$ during the 3 rd period.

\subsection{Patterns of the DST}

Table 3 gives us patters of resistance to the 4 molecules tested with DST and (C) the different proportions of the combinations of the resistances between the 4 molecules tested divided by period.

Patients with a sensitivity test of at least Rifampicin and Isoniazid were at 2031. The proportion of those with at least first-line resistance was 1205 or $59.3 \%$ [ $95 \%$ CI 57.2 - 61.4], of which 1018 or $50.1 \%$ [CI 95\% $47.9-52.3$ ] for INH, 926 or 45.6\% [95\% CI 43.4 - 47.8] for Rifampicin, 1014 or $49.9 \%$ [IC 95\% 47.8 - 52.1] for Ethambutol and 727 or $35.8 \%$, [95\% CI 33.7 - 37.9] for Streptomycin. Resistance combinations showed a proportion of MDR-TB at $42.8 \%$ [95\% CI 38.4 - 47.8]. 
Table 3. Laboratory results containing (A) Availability of tests (B) patterns of resistance to first line drugsand (C) proportion of RR-/MDR-TB divided by period.

\begin{tabular}{|c|c|c|c|c|c|c|c|}
\hline & Period 1 & Period 2 & Period 3 & Period 4 & Period 5 & \multicolumn{2}{|c|}{ All Periods } \\
\hline \multicolumn{8}{|c|}{ A. Availability of tests for presumptive patients } \\
\hline Test performed & $\mathrm{n}=595(\%)$ & $\mathrm{n}=1023(\%)$ & $\mathrm{n}=1446(\%)$ & $\mathrm{n}=2148(\%)$ & $\mathrm{n}=1300(\%)$ & \multicolumn{2}{|c|}{$\mathrm{N}=6512(\%)$} \\
\hline Culture solid L-J & $594(99.8)$ & $1021(99.8)$ & $1141(78.9)$ & $1226(57.1)$ & $694(53.4)$ & \multicolumn{2}{|c|}{$4676(71.8)$} \\
\hline Drug sensitivity test & $434(72.9)$ & $588(57.5)$ & $397(27.5)$ & $432(20.1)$ & $180(13.8)$ & \multicolumn{2}{|c|}{$2031(31.2)$} \\
\hline GeneXpert MTB/RIF & - & - & $36(2.5)$ & $668(31.1)$ & $608(46.8)$ & \multicolumn{2}{|c|}{$1312(26.8)$} \\
\hline $\begin{array}{l}\text { Culture LJ and/or } \\
\text { Xpert MTB/RIF }\end{array}$ & $594(99.8)$ & $1021(99.8)$ & $1166(80.6)$ & $1701(79.2)$ & $925(71.2)$ & \multicolumn{2}{|c|}{$5407(83.0)$} \\
\hline \multicolumn{8}{|c|}{ Patterns of resistance to first line anti-TB drugs } \\
\hline Profile of resistance & $\mathrm{n}=434(\%)$ & $\mathrm{n}=588(\%)$ & $\mathrm{n}=397(\%)$ & $\mathrm{n}=432(\%)$ & $\mathrm{n}=180(\%)$ & $\mathrm{N}=2031(\%)$ & [IC95\%] \\
\hline Sensible to all drugs & $129(29.7)$ & $223(37.9)$ & $256(64.5)$ & $147(34.0)$ & $71(39.4)$ & $826(40.7)$ & {$[38.6-42.8]$} \\
\hline Resistance to $\mathrm{H}$ & $278(64.1)$ & $306(52)$ & $124(31.2)$ & $215(49.8)$ & $95(52.8)$ & $1018(50.1)$ & {$[47.9-52.3]$} \\
\hline Resistance to R & $243(56.0)$ & $289(49.1)$ & $90(22.7)$ & $208(48.1)$ & $96(53.3)$ & $926(45.6)$ & {$[43.4-47.8]$} \\
\hline Resistance to E & $241(55.5)$ & $306(52)$ & $120(30.2)$ & $242(56.0)$ & $105(58.3)$ & $1014(49.9)$ & {$[47.8-52.1]$} \\
\hline Resistance to S & $216(49.8)$ & $219(37.2)$ & $97(24.4)$ & $123(28.5)$ & $72(40.0)$ & $727(35.8)$ & {$[33.7-37.9]$} \\
\hline Total mono resistance & $26(5.9)$ & $46(7.8)$ & $16(4.0)$ & $64(14.8)$ & $3(1.7)$ & $155(7.6)$ & {$[5.8-10.2]$} \\
\hline Mono resistance to $\mathrm{H}$ & $12(2.8)$ & $7(1.2)$ & $6(1.5)$ & $5(1.2)$ & $0(0)$ & $30(1.5)$ & {$[1.0-2.1]$} \\
\hline Mono resistance to $\mathrm{R}$ & $3(0.7)$ & $4(0.7)$ & $0(0)$ & $6(1.4)$ & $1(0.6)$ & $14(0.7)$ & {$[0.4-1.2]$} \\
\hline Mono resistance to $\mathrm{E}$ & $5(1.2)$ & $26(4.4)$ & $8(2.0)$ & $52(12)$ & $2(1.1)$ & $93(4.6)$ & {$[3.8-5.6]$} \\
\hline Mono resistance to $S$ & $6(1.4)$ & $9(1.5)$ & $2(0.5)$ & $1(0.2)$ & $0(0)$ & $18(0.9)$ & {$[0.6-1.4]$} \\
\hline $\begin{array}{l}\text { Total multidrug } \\
\text { resistance }\end{array}$ & $229(52.8)$ & $271(48.6)$ & $85(21.4)$ & $193(44.7)$ & $91(50.6)$ & $869(42.8)$ & {$[38.4-47.8]$} \\
\hline $\mathrm{H}-\mathrm{R}$ & $12(2.8)$ & $13(2.2)$ & $6(1.5)$ & $15(3.5)$ & $2(1.1)$ & $48(2.4)$ & {$[1.8-3.1]$} \\
\hline H-R-E & $42(9.7)$ & $77(13.1)$ & $9(2.3)$ & $65(15)$ & $26(14.4)$ & $219(10.8)$ & {$[9.5-12.2]$} \\
\hline H-R-S & $22(5.1)$ & $21(3.6)$ & $6(1.5)$ & $15(3.5)$ & $0(0)$ & $64(3.2)$ & {$[2.5-4.0]$} \\
\hline H-R-E-S & $153(35.3)$ & $160(27.2)$ & $64(16.1)$ & $98(22.7)$ & $63(35)$ & $538(26.5)$ & [24.6 - 28.5] \\
\hline Total poly resistance & $50(11.5)$ & $48(8.2)$ & $40(10.1)$ & $28(6.5)$ & $15(8.3)$ & $181(8.9)$ & {$[6.4-12.8]$} \\
\hline H-E & $10(2.3)$ & $12(2.0)$ & $14(3.5)$ & $13(3.0)$ & $2(1.1)$ & $51(2.5)$ & {$[1.9-3.3]$} \\
\hline $\mathrm{H}-\mathrm{S}$ & $10(2.3)$ & $5(0.9)$ & $3(0.8)$ & $1(0.2)$ & $1(0.6)$ & $20(1.0)$ & {$[0.6-1.5]$} \\
\hline H-E-S & $17(3.9)$ & $11(1.9)$ & $16(4.0)$ & $3(0.7)$ & $1(0.6)$ & $48(2.4)$ & {$[1.8-3.1]$} \\
\hline E-S & $2(0.5)$ & $6(1)$ & $2(0.5)$ & $2(0.5)$ & $3(1.7)$ & $15(0.7)$ & {$[0.5-1.2]$} \\
\hline R-E & $5(1.2)$ & $7(1.2)$ & $1(0.3)$ & $6(1.4)$ & $4(2.2)$ & $23(1.1)$ & {$[0.8-1.7]$} \\
\hline R-S & $0(0)$ & $1(0.2)$ & $0(0)$ & $0(0)$ & $0(0)$ & $1(0.1)$ & {$[0.0-0.3]$} \\
\hline R-E-S & $6(1.4)$ & $6(1)$ & $4(1.0)$ & $3(0.7)$ & $4(2.2)$ & $23(1.1)$ & {$[0.8-1.7]$} \\
\hline \multicolumn{8}{|c|}{ C. The proportion of RR-/MDR-TB versus non RR-/MDR-TB } \\
\hline & $\mathrm{n}=434(\%)$ & $\mathrm{n}=588(\%)$ & $\mathrm{n}=426(\%)$ & $\mathrm{n}=964(\%)$ & $\mathrm{n}=739(\%)$ & $\mathrm{N}=3151(\%)$ & [IC 95\%] \\
\hline RR-/MDR-TB & $243(56.0)$ & $289(49.2)$ & $97(22.8)$ & $434(45.0)$ & $501(67.8)$ & $1564(49.6)$ & {$[47.9-51.4]$} \\
\hline Non RR-/MDR-TB & $191(44.0)$ & $299(50.8)$ & $329(77.2)$ & $530(55.0)$ & $238(32.2)$ & $1587(50.4)$ & {$[48.6-52.1]$} \\
\hline
\end{tabular}

E: Ethambutol. H: isoniazid. rifampicin and S: streptomycin. 
Among the 1312 patients subjected to Xpert. The MTB were detected at 1207 (91\%).

Taking into account both tests. The proportion of RR-/MDR-TB was $49.6 \%$ [95\% CI 47.9 - 51.4] accounting for almost half of the patients who tested mycobacterium in sputum.

Among the results contaminated with culture 62 revealed the presence of MTB GeneXpert 56 with RR-TB (84\%). Of the negative results in solid culture 265 were MTBs, of which 236 with RR (88\%). The agreement between RR and MDR-TB was 99\% (75TB-MR/76RR-TB).

\subsection{Proportion by Type of Presumptives}

Figure 4 gives a representation of the proportions of RR-/MDR-TB and non $\mathrm{RR}-/ \mathrm{MDR}-\mathrm{TB}$ by type of presumptive seen in descending order.

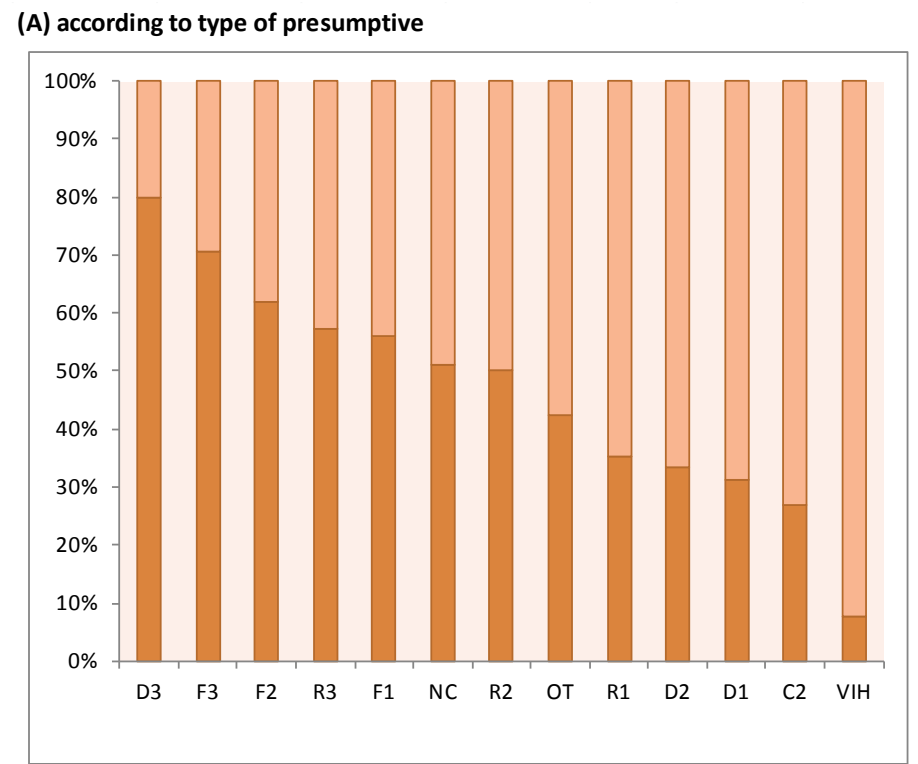

(B) according to number of previous episode and outcome at the last treatment

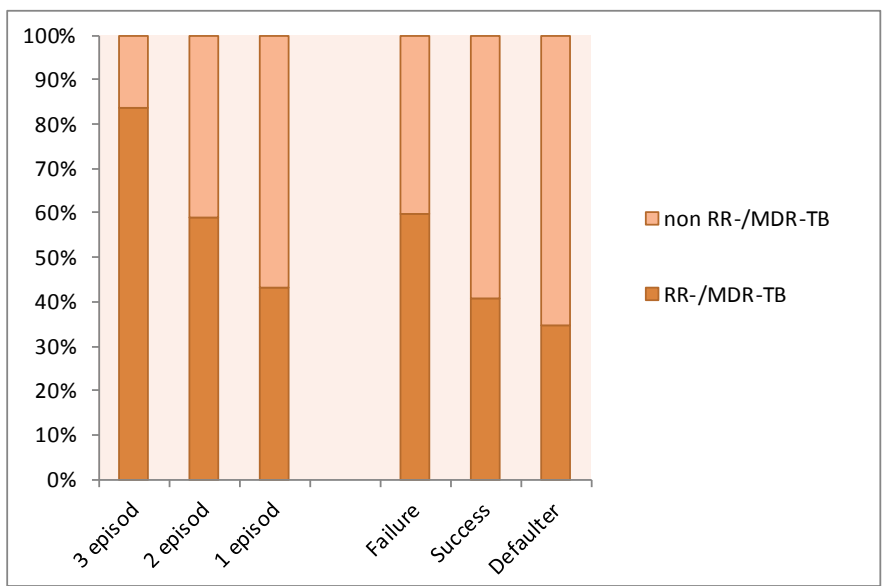

Figure 4. The proportions of RR-/MDR-TBaccording (A) to type of presumptive and (B) number of previous treated episode and outcome at the last treatment. 
The ranking in this chart is done in descending order of proportions of $\mathrm{MDR} / \mathrm{RR}$ - TB in each group.

In this study were evaluated some factors associated with the presence and diagnosis of MDR-TB in Table 4.

In logistic regression analysis. An age less than or equal to 35 years was 1.5 times more likely to have MDR-TB. Regarding treatment outcomes at the last episode, a treatment failure and treatment interruption was respectively 3.5 and 2.2 times more likely to have MDR-TB than a patient with therapeutic success. For DST on solid-medium culture diagnosis, a 7-day specimen delivery delay increases the chances of having MDR-TB by 2.5 and 1.7 times when the delay between the collection and the sample processing is less than 2 weeks.

The higher numbers of previous episodes among previously treated cases, although associated in bivariate analysis, did not remain associated in multivariate analysis.

Table 4. Factors associated with the diagnosis of MDR-TB.

\begin{tabular}{|c|c|c|c|c|c|c|}
\hline \multirow{2}{*}{ Variable } & \multicolumn{3}{|c|}{ Bivariate analysis } & \multicolumn{3}{|c|}{ Multivariate analysis } \\
\hline & \multicolumn{2}{|c|}{ OR (95\% CI) } & $\mathrm{p}$ & \multicolumn{2}{|c|}{ Adjusted OR (95\% CI) } & \multirow[t]{2}{*}{$\mathrm{p}$} \\
\hline \multicolumn{6}{|l|}{ Sex } & \\
\hline - Female & & 1 & & & 1 & \\
\hline - male & 1.072 & (0.898 to 1.281$)$ & 0.441 & 1.095 & (0.852 to 1.408$)$ & 0.479 \\
\hline \multicolumn{7}{|c|}{ Age of the patient } \\
\hline - 35 years & & 1 & & & 1 & \\
\hline - $\leq 35$ years & 1.507 & (1.221 to 1.856$)$ & 0.000 & 1.397 & (1.088 to 1.794$)$ & 0.009 \\
\hline \multicolumn{7}{|c|}{ outcome at the last episode } \\
\hline - Success & & 1 & & & 1 & \\
\hline - failure & 2.092 & (1.066 to 4.106$)$ & 0.032 & 3.508 & (1.196 to 10.285$)$ & 0.022 \\
\hline - defaulter & 2.341 & (1.858 to 2.950$)$ & 0.000 & 2.223 & (1.575 to 3.137$)$ & 0.000 \\
\hline \multicolumn{7}{|c|}{ Number of previous episodes of TB } \\
\hline - one & & 1 & & & 1 & \\
\hline - Two or more & 2.043 & (1.299 to 3.214$)$ & 0.002 & 1.413 & $(0.959$ to 2.082$)$ & 0.080 \\
\hline \multicolumn{7}{|l|}{ Origin } \\
\hline - Rural & & 1 & & & 1 & \\
\hline - Urban & 1.015 & $(0.813$ to 1.475$)$ & 0.552 & 1.01 & $(0.589$ to 1.732$)$ & 0.971 \\
\hline \multicolumn{7}{|c|}{ Delay (1) of the delivery of samples* } \\
\hline - $>7$ days & & 1 & & & 1 & \\
\hline - $\leq 7$ hours & 1.451 & (1.121 to 1.878$)$ & 0.005 & 2.452 & $(1.345$ to 4.469$)$ & 0.003 \\
\hline \multicolumn{7}{|c|}{ Delay (2) of the realization of the culture * } \\
\hline - $>10$ days & & 1 & & & 1 & \\
\hline - $\leq 10$ hours & 1.832 & $(1.454$ to 2.311$)$ & 0.000 & 1.375 & $(0.960$ to 1.970$)$ & 0.083 \\
\hline \multicolumn{7}{|c|}{ Time (3) between the picking of sputum and the test completion * } \\
\hline - >14 days & & 1 & & & 1 & \\
\hline - $\leq 14$ hours & 1.843 & (1.420 to 2.391$)$ & 0.000 & 1.672 & (1.092 to 2.562$)$ & 0.018 \\
\hline
\end{tabular}

Delay (1) represents the number of days between the sputum collection and shipment to the laboratory. The delay (2) is the number of the days between the arrival of the samples at the laboratory the realization of the culture and the delay (3) is the total of the days between the picking of sputum and the test completion. For these different delays, we have considered the average of the days as reference point. 


\section{Discussion}

The present study analyzed the proportion of RR-/MDR-TB among presumptives in the DRC and some factors associated with the diagnosis of MDR-TB during 10 years of surveillance. Eight thousand eight (8008) patients were identified in the laboratory for a diagnosis of TB and MDR-TB, of which 6512 (81.3\%) were known to be presumptive of MDR-TB. For 1105/6512 (17.0\%) patients, the results were either not found or unrealized. The analysis were not performed for a variety of reasons, the most common being the loss of samples, empty spittoons or unusable samples.

This study counted a total of 13 presumptive groups. Previously treated patients accounted for the majority (95\%), new patients were poorly represented (2\%), and the remainder was the HIV seropositive group without patient's history details. Retreatment failures formerly known as chronic cases constituted the majority group (30\%). They have long been considered the most at risk and therefore a priority [1] [16] [22]. These failures, which included three-quarters of patients in the first period, accounted for only one-fifth in the last few years of the study period. At the same time as their proportion fell in a linear manner, so was the absolute number. This could be explained by the fact that the patients in retreatment are detected more and earliest with the integration of F1 and R1. Patients failing and relapsing after a first episode did not become actual targets until later, as their registration increased as shown in Figure 2. This situation has also been found in Bangladesh, with time there have been fewer and fewer suspected MDR-TB cases with multiple episodes [8]. Comparing the time of issuance of the guidelines with their application, this suggests a delay that may suggest that many patients have not been screened for MDR-TB. These patients were therefore not spared the prolonged use (of the additional drug pressure) of the RMP and INH (genetic mutation determining factor) responsible for the genesis of drug resistance [2] [22].

The analysis showed that HIV patients as targets noted in the 2nd period, were recognized at the beginning of the implementation of the response against MDR-TB. But their application in a context of inadequate HIV testing and an under-mobilized population has not been facilitated. This deficiency is known in several reports covering the period. The number of TB patients tested in the DRC has long been below 50\% [23] [24]. However, some patients have been registered and have benefited from screening. Improving global HIV-TB and TB-HIV collaborative activities, which advocate for systematic TB-screening of HIV-positive people, provides more comprehensive information [25]. HIV patients have been considered presumptive to carry TB for several years and their analysis in this setting will increase confounders. They are analyzed as a separate group and give some information, although insufficient. The Correct assessment of co-infection indicators would be more accurate with more complete data. The most appropriate research for TB and MDR-TB in HIV patient is the combination of genotypic testing and other methods in accordance with recent guidelines with more elaborate algorithms [25] [26]. 
The comparison between the numbers of patients registered for retreatment during the period and those whose samples were sent to the laboratory shows that the proportions are very small (less than $10 \%$ ) as shown in previous analyze [5] [23] [27]. The limits are numerous as described by Nourzad [28] who showed that in all the countries studied, less than 50\% of MDR-TB presumptive had a DST and a little more than half in a study in Peru [29]. The deficit related to sample transportation, cost, logistics and the preparation of the package to be transported guaranteeing safety according to the ISO standards [7] [19] explains why the majority of the samples come from the urban environments or close to the laboratory, the case of Kinshasa, Kongo-Central or near an airport.

The city of Kinshasa is the home of the main screening laboratory. The proportion is more than half of the cases. This situation is encountered in most countries with few laboratory units that can screen for MDR-TB in developing countries [26] [30]. In some African countries, an organized system of sample transport has been put in place using all the ways (road, rail and air) where the infrastructure permits. The resolution of the sample transfer problem in the DRC remains a major challenge despite recent efforts, screening is still low [5]; this big issue requires multisectoral and permanent measures that should be properly applied and assessed.

This analysis showed an incomplete availability of phenotypic sensitivity tests that decreased with time and the number of samples, and only a part of patients have access to a quality diagnosis before the advent of gambling rapid and molecular test [3] [31] [32]. In the present study more than $80 \%$ of patients had a laboratory result; this influences the level of validity because it is an optimal percentage for evaluations of routine monitoring [6] [28]. The spread of the rapid test has the downside of the use of conventional methods, which become reserved for the results that have detected a RR-TB which we necessarily want to have a more complete resistance profile including the detection of extensively drug- resistance (XDR-TB) [26]. This decline was marked during the 3rd period of the study.

The last two periods were dominated by the use of the Xpert MTB/RIF test, although the test detects only the resistance to RMP. These performances have been described in several studies at the global and national level [3] [33], both for the detection of MTB and for the diagnosis of DR-TB, through the RR-TB recognized as a proxy to MDR-TB. The Xpert-MTB/RIF test detected RR-TB in 56 formerly contaminated specimens and $236 \mathrm{RR}-\mathrm{TB}$ in those that were negative for culture on solid media. This is largely due to the high sensitivity of the Xpert MTB/RIF test.

In this study, the resistance pattern to 4 molecules listed in Table 3 has shown a proportion of MDR-TB of $49.6 \%$ in a population with a large majority of patients repeatedly exposed to RMP and INH. The number of resistances was associated with the number of previous regimens by amplification phenomenon, which is described in other studies [8] [34] [35]. 
This proportion of MDR-TB is divergent from that of $17 \%$ found by the drug resistance survey conducted in the DRC between 2015 and 2016 mentioned in the WHO Report [5]. This proportion is also higher than that found among the cases already treated by Van Deun et al. in Bangladesh. These authors had found a percentage of MDR-TB of $11.7 \%$ (range 8.1 - 23.5) among the failures and relapses at the beginning of the retreatment [8]. In the context of Bangladesh at the time, Category 1 or the initial treatment was predominantly made of 2 RHEZ/6HT (containing thiacetazone in place of ethambutol) for this sample of patients. This regimen is less inducing MDR-TB than those used in the DRC made of 2 RHEZ/6EH (until 2007) and 2 RHEZ/4RH. An investigation more than 10 years after the introduction of the $2 \mathrm{RHEZ} / 4 \mathrm{RH}$ regimen in Bangladesh showed a higher rate of MDR-TB among the previously treated cases [34].

The systematic review done in Ethiopia (2017) showed higher proportions than the present study among the cases previous treated, here the prevalence of MDR-TB in new patients is associated with this rate, was 6 times higher than that found in the DRC [35]. In this same review of several studies, they showed that in the same region, the resistance profile and the prevalence of MDR-TB could vary greatly. Several determinants were considered, the previous regime and its duration, regularity of treatment and socioeconomic factors [35].

Compared to previous studies conducted in the DRC, the difference with this survey can be in the sampling. In the 2015-2016 national survey identified above that reported $17 \%$ of RR-/MDR-TB among previously treated cases [5], values lower than those described in this study. In this prevalence survey the cases already treated represent only $10 \%$ of the total sample of patients and the majority of which were retreatment cases. Another element that would support this difference is that the distribution did not take into account the burden of TB disease in the national territory. The selected clusters between rural and urban areas were equitably distributed when they were not. In this analysis, high -burden provinces are the most represented.

The percentage of MDR-TB confirmed is still under discussion as to the question of the denominator for this indicator, all registered presumptives or essentially patients with a laboratory result. These conditions expose the risk of either minimizing or overestimating the magnitude of the problem if an adjustment factor is not applied [6] [8] [22]. The use of the proportions on the present tests is more precise, it is used to extrapolate the results on a larger population if one wants to estimate the burden of acquired MDR-TB [14] [22]. The burden of acquired MDR-TB will consider the rate of MDR-TB to be applied to each group of presumptives, adjusted for factors related to undetected cases, either due to insufficient health coverage or poor system performance [5].

In the present study patients sensitive to all 4 molecules tested represented $40 \%$ of cases; this requires careful interpretation especially when the therapeutic history is known. It is possible that no mutation occurs despite the conditions of therapeutic insufficiency. Indeed, the appearance of a mutation responds to the random mode [36]. Since mutation is a phenomenon that depends on chance, 
the probability of a mutation occurring is complex and difficult to apply to a single individual. Another way of explanation would be the exogenous reinfection by a strain totally sensitive to the classical test; this notion is well exploited in the hypotheses studying the dissemination of communicable diseases [2] [12] [22].

The classical test which is based on the comparison of the conventionally established proportion (resistant strains/susceptible strains) shows its limits. It is the dominant strain that leads the pattern. However, there are other strains carrying resistant mutation genes that remain hidden. This is also applies to mono-resistance cases which accounted for $8 \%$ to $10 \%$ of patients and careful interpretation should applied [8] [18] [37].

The logistic regression analysis showed that age under 35 was associated with MDR-TB, and the risk is twice that of those over 35 years of age. This corroborates the situation found in a previous study in Kinshasa [1] [7] [38], Bangladesh [8] [34] and Ethiopia [30] [35] which showed that although the average age of patients with TB and MDR-TB is increasing, it still affects the youngest age groups.

Regarding the history of $\mathrm{TB}$, multivariate logistic regression analysis showed that the possibility of finding MDR-TB was greater for treatment failures, the risk was twice greater than those who default in the past and four times more than those who had therapeutic success, which is consistent with observations from other studies in which the genesis of MDR-TB was first associated with failure and relapse and lost to follow up [17] [22] [39] [40]. For the last two groups the positions could vary. Indeed, in the group of defaulter's patients, there are patients with sputum that has been negativated or converted to bacillary load. The causes of the interruptions are various, sometimes due to a bad evolution and sometimes follow a good clinical evolution during the continuation phase when the patient resumes his usual activities [41] [42].

This continuous surveillance based on routine testing also showed that both times of sputum delivery and screening tests have an influence on the diagnosis of drug resistance when using culture; this is not confirmed for molecular testing. The faster a sample is transported and processed in the laboratory, the more satisfactory the results. This has also been described elsewhere [6] [26] [43]. Culture requires living germs whereas molecular tests are based on the presence of genetic material even though germs have lost viability. It is therefore imperative to strengthen the transportation of specimens and imperatively achieve the culture which remains the accurate one. We therefore do not encourage the abandonment of culture and DST at the advent of molecular tests, but rather to move towards culture on liquid media and tests to detect the viability of germs using algorithms combining all these methods [26].

\subsection{Limitations}

Some limitations are recognized by the authors of this work; those related to the 
analysis of historical cohorts that are often subject to information biases due to different registration methods caused by the change of directives. The corrections were made by verifying and comparing the data with MDR-confirmed cases registers which contained more details about the patients. The number of results not found has certainly reduced the accuracy of the calculations.

\subsection{Strengths}

The routine monitoring over a long period of time reflects variations over time and provides more accurate averages. It is one the first study of its kind.

\section{Conclusion}

The proportion of RR-/MDR-TB among the presumptive has represented higher rate than those estimated generally. The NTP should improve patient follow-up to reduce TB treatment failures and defaulting; also, while increasing the use of molecular tests, they should reduce sample delivery times when using culture and DST concomitantly.

\section{Contributions of the Authors}

SBF, principal investigator;

SBF, JKN, PKK for the design of the study;

PKK, ESK, SBF for data analysis;

PMS, SBF, coordinated the collection of data;

MKB, HSN, JDD, JKN, PKK for proofreading and editing of the final form of article.

\section{Acknowledgements}

Thanks to the officers of the National Laboratory of reference especially to $\mathrm{Bu}$ teka JF, and Simelo JP, the Head of National Tuberculosis Programme/DRC, provincial coordinators, nurses of health centers and technicians of field laboratories and Professor Georges Mpwate for revising the document.

\section{Conflicts of Interest}

The authors declare no conflicts of interest regarding the publication of this paper.

\section{References}

[1] World Health Organization (2008) Guidelines for the Programmatic Management of Drug-Resistant Tuberculosis. Emergency Update. Geneva. http://whqlibdoc.who.int/publications/2008/9789241547581_eng.pdf

[2] Johnson, R., Streicher, E.M., Louw, G.E., Warren, R.M., Helden, P.D. and Victor, T.C. (2005) Drug Resistance in Mycobacterium Tuberculosis. Current Issues in Molecular Biology, 8, 97. http://www.cimb.org

[3] Steingart, K.P., Sohn, H., Schiller, I., Kloda, L.A., Boehme, C.C., Pai, M. and Dendukuri, N. (2013) Xpert ${ }^{\oplus}$ MTB/RIF Assay for Pulmonary Tuberculosis and Rifampi- 
cin Resistance in Adults. Cochrane Database of Systematic Reviews, 1, CD009593. https://doi.org/10.1002/14651858.CD009593.pub2

[4] World Health Organization (2017) Guidelines for the Treatment of Drug Susceptible Tuberculosis and Patient Care. 2017 Update, Geneva, 1-73. http://www.who.int/tb/publications/2017/dstb_guidance_2017/en/

[5] World Health Organization (2017) Global Tuberculosis Report 2017. GTB, Geneva, WHO/HTM/TB/2017.

[6] Dean, A. and Zignol, M. (2015) Guidelines for Surveillance of Drug Resistance in Tuberculosis. 5th Edition, WHO, Geneva, 1-73.

[7] Programme National de lutte contre la Tuberculose (2016) Guide technique de prise en charge de la Tuberculose multi résistance. Kinshasa. Ministère de Santé-RDC, 10-154.

[8] Van Deun, A., Hamid Salim, A., Rigouts, L., Rahman, M., Fissette, K. and Portaels, F. (2001) Evaluation de la lutte antituberculeuse par des tests de sensibilité périodiques ou en routine dans les cas traités antérieurement. International Journal of Tuberculosis and Lung Disease, 5, 329-338.

[9] Nkhoma, W.A.C. (2012) Multidrug-Resistant and Extensively-Resistant Tubercusosis in the African Region. The African Health Monitor Diseases Control. Communicable Diseases Control, 15, 22-29.

[10] World Health Organization (2018) Global Tuberculosis Report 2018. Geneva.

[11] Bisuta-Fueza, S., Kayembe-Kalambayi, P., Kabedi-Bajani, M., Situakibanza-Nani, T.H. and Kayembe-Ntumba, J.M. (2018) Tendances de la tuberculose pulmonaire bactériologiquement confirmée et issues thérapeutiques en République Démocratique du Congo: 2007-2017. Annals of African Medicine, 11, e2489-e2496.

[12] World Health Organization (2014) Companion Handbook to the WHO Guidelines for the Programmatic Management of Drug-Resistant Tuberculosis. WHO, Geneva, WHO/HTM/TB/2014.11.

[13] Zignol, M., Dean, A.S., Falzon, D., Gemert, W., Wright, A., Van Deun, A., Portaels, F., Laszlo, A., et al. (2016) Twenty Years of Global Surveillance of Antituberculosis-Drug-Resistance. The New England Journal of Medicine, 375, 1081-1089.

[14] Pavlenko, E., Barbova, A., Hovhannesyan, A., Tsenilova, Z., Slavuckij, A., Shcherbak-Verlan, et al. (2018) Alarming Levels of Multidrug-Resistant Tuberculosis in Ukraine: Results from the First National Survey. International Journal of Tuberculosis and Lung Disease, 22, 197-205.

[15] Programme National de lutte contre la Tuberculose (2016) Guide technique de prise en charge de la Tuberculose Programme Anti Tuberculeux intégré aux soins de Santé de base 5ème édition. Kinshasa, Ministère de la Santé.

[16] Misombo, A., Nguefack-Tsague, G., Kalla, G.M., Afane Ze, E., Kimpanga, D., Panda, T., Ilunga Kebela, Bisuta Fueza, S., Magazani, N. and Mbopi-Kéou, F.-X. (2016) Facteurs de risque de la tuberculose multi-résistante dans la ville de Kinshasa en République Démocratique du Congo. Pan Afrincan Medical Journal, 23, 1-11.

[17] Kayembe, J.M., Lukiana, T. and Bisuta, F.S. (2004) Tuberculose Chronique: Profil épidémiologique aux Cliniques Universitaires de Kinshasa (2000-2003). International Journal of Tuberculosis and Lung Disease, 11, S80.

[18] Kabedi, M.J., Kashongwe, M., Kayembe, J.M., Mumba Ngoyi, D., Mampas, I.P., Mbaya, P., Fissette, K., Verheagen, J., Porteals, F. and Muyembe, T.J. (2007) Résistance primaire de Mycobacterium tuberculosis aux antituberculeux à Kinshasa, République Démocratique du Congo. Bulletin de la Société de Pathologie Exotique, 100, 275-276. 
[19] World Health Organization (2012) Biosafety of Tuberculosis Laboratory. Geneva.

[20] World Health Organization (2011) Guidelines for the Programmatic Management of Drug-Resistant Tuberculosis. Geneva.

[21] World Health Organization (2014) Définitions et cadre de notification pour la Tuberculose-Révision 2013. Genève.

[22] Caminero, J.A. (2010) Multidrug-Resistant Tuberculosis: Epidemiology, Risk Factors and Case Finding. International Journal of Tuberculosis and Lung Disease, 14, 382-390.

[23] World Health Organization (2016) Global Tuberculosis Report 2016. Geneva.

[24] ONUSIDA. Rapport ONUSIDA 2016. Geneva.

[25] World Health Organization (2015) Collaborative HIV/TB Implementing Strategy. Geneva.

[26] Global Laboratory Initiative-Stop Tb Department (2017) Model Tuberculosis Diagnostic Algorithms. World Health Organization, Geneva.

[27] Kai, B. (2007) Barrier to Reaching the Target for Tuberculosis Control: Multidrug Resistance Tuberculosis. Bulletin of World Health Organ, 85, 387-394. https://doi.org/10.2471/BLT.06.035345

[28] Nourzad, S., Jenkins, H.E., Milstein, M. and Mitnick, C.D. (2017) Estimating the Global Burden of Multidrug-Resistant Tuberculosis among Prevalent Cases of Tuberculosis. International Journal of Tuberculosis and Lung Disease, 21, 6-11.

[29] Velásquez, G., Yagui, M., Cegielski, P., Asencios, L., Bayona, J., Cesar, B., Hector Yale, G., Suárez, C., Atwood, S., Contreras, C. and Shin, S. (2011) Targeted Drug-Resistance Testing Strategy for Multidrug-Resistant Tuberculosis Detection, Lima, Peru, 2005-2008. Emerging Infectious Diseases, 17, 3.

[30] Assefa, D., Seyoum, B. and Oljira, L. (2017) Determinants of Multidrug-Resistant Tuberculosis in Addis Ababa, Ethiopia. Infection and Drug Resistance. Open Access, 10, 209-213. https://www.dovepress.com/

[31] World Health Organization (2014) Xpert MTB/RIF Implementation Manual-Technical and Operational "How-to Practical Considerations". WHO, Geneva.

[32] Falzon, D., Mirzayev, F., Wares, F., Baena, I., Zignol, M., Linh, N., Weyer, K., Jaramillo, E., Floyd, K. and Raviglione, M. (2015) Multidrug-Resistant Tuberculosis around the World: What Progress Has Been Made? European Respiratory Society, 45, 150-160.

[33] Kabedi, B., Kayembe Ntumba, J.M., Kashongwe, M.Z., Bisuta Fueza, S., Mbaya Kalumba, P., Taba Kalulu, M. and Muyembe, T.J. (2017) GEN-XPERT ${ }^{\oplus}$ MTB/RIF Contribution in the Diagnosis of Pulmonary Tuberculosis with a Negative Bacilloscopy in Kinshasa (DRC). International Journal of Medical Reviews, 3, 160-163.

[34] Kamal, M.M., Hossain, M.A., Sultana, S., Begum, V., Haque, N., Ahmed, M.J., Rahman, M.A., Hyder, M.A., Hossain, Md.S., Rahman, M., Chowdhury, R.A., Aung, K.J.M., Islam, Md.A., Hasan, M.R. and Van Deun, A. (2015) Résistance aux médicaments antituberculeux au Bangladesh: Réflexions à partir de la première enquête nationale. International Journal of Tuberculosis and Lung Disease, 19, 151-156. https://doi.org/10.5588/ijtld.14.0200

[35] Weldegebreal, S. and Mebrahtu, T. (2017) Anti-Tuberculosis Drug Resistance in Ethiopia: Systematic Review. International Journal of Tuberculosis and Lung Disease, 21, 18-22. https://doi.org/10.5588/ijtld.16.0286

[36] McGrath, M., Gey van P., N.C., van Helden, P.D., Warren, R.M. and Warner, D.F. (2014) Mutation Rate and the Emergence of Drug Resistance in Mycobacterium tu- 
berculosis. Journal of Antimicrobial Chemotherapy, 69, 292-302. https://doi.org/10.1093/jac/dkt364

[37] Zignol, M., Van Gemert, W., Falzon, D., Sismanidis, C., Glaziou, P., Floyd, Ka. and Raviglione, M. (2012) Surveillance of Anti-Tuberculosis Drug Resistance in the World: An Updated Analysis, 2007-2010. Bulletin of the World Health Organization, 90, 111-119.

[38] Bisuta, F.S., Kashongwe, M.Z., Kayembe, N.J.M., Mbaki, N.C., Bahati, R.E., Simelo, K.J.P., Muyembe, T.J.J. and Bakaswa, N.G. (2006) Prise en charge de la tuberculose à bacilles multirésistants avec les antituberculeux de seconde ligne à Kinshasa: Résultats préliminaires. Congo Médical, 4, 504-512.

[39] Kritski, A.L., Rodrigues de Jesus, L., Andrade, M.K., Werneck-Barroso, E., Vieira, M.A., Haffner, A. and Riley, L.W. (1997) Retreatment Tuberculosis Cases. Factors Associated with Drug Resistance and Adverse Outcomes. Chest, 111, 1162-1167. https://doi.org/10.1378/chest.111.5.1162

[40] Lukoye, D., Ssengooba, W., Musisi, K.W., Kasule, G., Cobelens, F.G., Joloba, M. and Gomez, G.B. (2015) Variation and Risk Factors of Drug Resistant Tuberculosis in Sub-Saharan Africa: A Systematic Review and Meta-Analysis. BMC Public Health, 15, 1-13. http://www.biomedcentral.com https://doi.org/10.1186/s12889-015-1614-8

[41] Espinal, M.A., Kim, S.J., Suarez, P.G., Kam, K.M., Khomenko, A.G., Migliori, G.B., Baéz, J., Kochi, A., Dye, C. and Raviglione, M. (2000) Standard Short Course Chemotherapy for Drug-Resistance Tuberculosis. Treatment Outcomes in 6 Countries. Journal of American Medical Association, 283, 2537-2545. https://doi.org/10.1001/jama.283.19.2537

[42] Sberbaro, J. (2004) How Frequently Do Patients Stop Taking Treatment Prematurely? In: Toman's Tuberculosis Case Finding, Treatment and Monitoring, Questions and Answer, 2nd Edition, Geneva, 181-182.

[43] Banu, S., Rahman, M.T., Ahmed, S., Khatun, R., Ferdous, S.S., Hosen, B., Rahman, M.M., Ahmed, T., Cavanaugh, J.S. and Heffelfinger, J.D. (2017) Multidrug-Resistant Tuberculosis in Bangladesh: Results from a Sentinel Surveillance System. International Journal of Tuberculosis and Lung Disease, 21, 12-17. https://doi.org/10.5588/ijtld.16.0384 